\title{
Inhibition of TBK1 attenuates radiation-induced epithelial-mesenchymal transition of A549 human lung cancer cells via activation of GSK-3 $\beta$ and repression of ZEB1
}

Wen Liu', Yi-Juan Huang ${ }^{1}$, Cong Liu', Yan-Yong Yang, Hu Liu, Jian-Guo Cui, Ying Cheng, Fu Gao, Jian-Ming Cai and Bai-Long Li

Radiotherapy is an effective treatment method for lung cancer, particularly when the disease is at an advanced stage. However, previous researchers have observed that the majority of patients with conventional radiation therapy develop distant metastases and succumb to the disease. Thus, identifying and understanding novel pathways for the development of new therapeutic targets is a major goal in research on pulmonary neoplasms. Recent studies suggest that epithelial-mesenchymal transition (EMT) is the most important contributor to cancer metastasis. Induction of this complex process requires endogenously produced microRNAs; specifically, downregulation of the miRNA-200c causes an induction of EMT. We recently identified the tank-binding kinase-1 (TBK1) as a downstream effector of the miR-200c-driven pathway, but the biological function of TBK1 in EMT remains unknown. In this study, we tested whether TBK1 has a role in radiation-induced EMT and identified associated potential mechanisms. Human alveolar type II epithelial carcinoma A549 cells were irradiated with ${ }^{60} \mathrm{Co} \gamma$-rays. Western blotting revealed a time- and dose-dependent decrease in E-cadherin with a concomitant increase in vimentin after radiation, suggesting that the epithelial cells acquired a mesenchymal-like morphology. TBK1 siRNA significantly inhibited radiationinduced suppression of the epithelial marker E-cadherin and upregulation of the mesenchymal marker vimentin. The invasion and migratory potential of lung cancer cells upon radiation treatment was also reduced by TBK1 knockdown. Furthermore, radiation-induced EMT attenuated by TBK1 depletion was partially dependent on transcriptional factor ZEB1 expression. Finally, we found glycogen synthase kinase-3 $\beta$ (GSK-3 $\beta)$ is involved in regulation of radiation-induced EMT by TBK1. Thus, our findings reveal that TBK1 signaling regulates radiation-induced EMT by controlling GSK-3 $\beta$ phosphorylation and ZEB1 expression. TBK1 may therefore constitute a useful target for treatment of radiotherapy-induced metastasis diseases.

Laboratory Investigation (2014) 94, 362-370; doi:10.1038/labinvest.2013.153; published online 27 January 2014

KEYWORDS: epithelial-mesenchymal transition; GSK-3 $\beta$; metasatsis; radiation; TBK1; ZEB1

Lung cancer is one of the most difficult cancers to cure and its mortality rates are the highest among all cancer worldwide. $^{1,2}$ In 2008 , lung cancer had replaced liver cancer as the first cause of death among people with malignant tumors in China. ${ }^{3}$ It is generally recognized that much scientific advancements such as surgery therapy, radiotherapy, and chemotherapy have been made in the area of lung cancer research and with much improvement in the application of these techniques, the chances of disease-free survival of lung cancer survivors have increased over last few decades. ${ }^{4,5}$ Unfortunately, there are many challenges in managing lung cancer patients. For example, although radiotherapy has been classically viewed to exert its therapeutic effect by killing tumor cells, emerging clinical studies suggest that patients show different responses to this therapy: some are cured, whereas others are prone to relapse and distant metastasis

Department of Radiation Medicine, Faculty of Naval Medicine, Second Military Medical University, Shanghai, PR China

Correspondence: Dr B-L Li, PhD or Dr J-M Cai, PhD, Department of Radiation Medicine, Faculty of Naval Medicine, Second Military Medical University, 800, Xiangyin Road, 200433, Shanghai, PR China.

E-mail: libailong2013@163.com or jianmingcai2003@163.com

${ }^{1}$ These authors contributed equally to this paper.

Received 6 August 2013; revised 13 September 2013; accepted 21 October 2013 
because of radioresistance. Besides, increasing radiation doses for treating radioresistant malignant cells was followed by inevitable toxic effects for normal tissues. ${ }^{6-8}$

Previous researchers have observed that the majority of patients with locally advanced, unresectable lung cancer treated with conventional radiation therapy develop distant metastases and succumb to the disease. ${ }^{9}$ Metastasis is the most important contributor to the mortality of patients with cancer. $^{10,11}$ The pathogenesis of cancer metastasis involves increased cell invasion, loss of cellular adhesion, survival in the circulation, entry into new tissue, and eventual colonization of distant organs. ${ }^{12}$ More recently, a conception called epithelialmesenchymal transition (EMT) has a central role in metastasis and has become the subject of intense investigation.

EMT is a complex biological process by which differentiated epithelial cells undergo remarkable morphological changes and characterized by a transition from epithelial cobblestone phenotype to elongated fibroblastic phenotype. The completion of an EMT is signaled by the downregulation of epithelial molecular markers such as E-cadherin and upregulation of mesenchymal molecular markers such as vimentin and $\mathrm{N}$-cadherin. ${ }^{13,14} \mathrm{EMT}$ is governed by a variety of regulatory networks and triggered by extracellular stimuli, such as transforming growth factor- $\beta$ (TGF- $\beta$ ), hypoxia, and radiation. ${ }^{15}$ Previous findings indicate that ionizing radiation (IR) is able to affect abundance of proteases and transcription factors involving in multiple signaling pathways participating in this complex pathogenesis. ${ }^{16-18}$ Thus, utilizing this knowledge and identifying novel pathways for therapeutic targets is the first goal in research on pulmonary neoplasms.

Tank-binding kinase-1 (TBK1), a new member of the inhibitor $\kappa \mathrm{B}(\mathrm{I} \kappa \mathrm{B})$ kinase-related kinase family, participates in inflammatory pathways related to activation of nuclear factor(NF)- $\kappa \mathrm{B}^{19}$ and has an essential role in Toll-like receptor- and retinoic acid-inducible gene-I-mediated induction of type I interferon (IFN- $\alpha / \beta)$ and host antiviral responses. ${ }^{20}$ We recently identified TBK1 is a downstream effector of the miR-200c-driven pathway. ${ }^{21}$ Consistently, researches have shown that miR-200c inhibited EMT by directly targeting ZEB1 and ZEB2. ${ }^{22}$ However, the detailed biological role of TBK1 in EMT remains largely unknown.

As the downstream of miR-200c signaling, we hypothesized a role for this regulator in radiation-induced EMT. Here, we elucidate a new EMT pathway mediated by TBK1 that leads to the repression of ZEB1 expression via the activation of glycogen synthase kinase- $3 \beta$ (GSK-3 $\beta$ ), which has been previously implicated in certain features of radiationinduced EMT. ${ }^{23}$ Increasing understanding of the role TBK1 in radiation-induced EMT will pave the way for more effective treatments in radiotherapy.

\section{MATERIALS AND METHODS}

\section{Cell Culture and Treatment}

Human alveolar type II epithelial carcinoma cell line A549 was obtained from the American Type Culture Collection
(Manassas, VA, USA) and routinely maintained in Dulbecco's modified Eagle medium (DMEM; PAA Laboratories) with $10 \%$ fetal bovine serum (PAA Laboratories) at $37^{\circ} \mathrm{C}$ in an atmosphere of $5 \% \mathrm{CO}_{2}$. For experiments with GSK-3 $\beta$ inhibitors, cells were treated with $10 \mu \mathrm{M}$ SB216763 for $16 \mathrm{~h}$ before irradiation. ${ }^{24,25}$

\section{Irradiation}

Cells were treated with $8 \mathrm{~Gy}$ of $\gamma$-rays irradiation using a ${ }^{60} \mathrm{Co}$ irradiator(Faculty of Naval Medicine, Second Military Medical University, China) at a dose rate of $3.64 \mathrm{~Gy} / \mathrm{min}$. All irradiations were performed at room temperature.

\section{Transient Transfection Assays}

Lipofectamine 2000 (Invitrogen, Carlsbad, CA, USA) was used for the RNA interference assay according to the manufacturer's instructions.

A549 cells were prepared at a density of $2 \times 10^{5}$ cells per well into a six-well plate with $2 \mathrm{ml}$ DMEM containing $10 \%$ fetal bovine serum and incubated until they reached $30-50 \%$ confluence. The confluent cells were transfected with 100 pmol of siRNA using $5 \mu \mathrm{l}$ of lipofectamine 2000 transfection reagent. After a $6 \mathrm{~h}$ incubation at $37^{\circ} \mathrm{C}$ in a $5 \% \mathrm{CO}_{2}$ humidified incubator, the serum-free medium was discarded and replaced with complete medium. Cells were then irradiated with a dose of $8 \mathrm{~Gy}$ of ${ }^{60} \mathrm{Co} \gamma$-rays at $24 \mathrm{~h}$ posttransfection, and cell lysates were harvested at the required time point for analysis.

\section{Western Blot Analysis}

Cellular proteins were obtained by using Proteo-JETTM Mammalian Cell Lysis Reagent (Thermo). Lysate proteins were mixed with an equal volume of loading buffer, denatured by heating at $100^{\circ} \mathrm{C}$ for $10 \mathrm{~min}$, and then separated on a 10 or $12 \%$ polyacrylamide minigel. The proteins were transferred to nitrocellulose membrane by wet-transfer, followed by blocking with $10 \%$ non-fat milk, and then incubated with primary antibodies overnight at $4{ }^{\circ} \mathrm{C}$. The primary antibodies were washed in Tris-buffered saline, pH7.5, supplemented with $0.1 \%$ Tween- 20 and then incubated with anti-rabbit IgG horseradish peroxidaseconjugated secondary antibody (Cell Signaling Technology, Danvers, MA, USA) for $2 \mathrm{~h}$ at room temperature. Finally, the signals of protein bands were detected with an ECL system (Thermo) according to the manufacturer's instructions.

\section{Immunofluorescence Staining}

Cells grown in a six-well chamber were transfected with TBK1-specific or control siRNA. $24 \mathrm{~h}$ after transfection, cells were irradiated with $8 \mathrm{~Gy}$ of ${ }^{60} \mathrm{Co} \gamma$-rays and were seeded into a 24-well chamber after $48 \mathrm{~h}$. $24 \mathrm{~h}$ later cells were washed with PBS, fixed in $4 \%$ paraformaldehyde for $1 \mathrm{~h}$ and permeabilized with $0.1 \%$ Triton $\mathrm{X}-100$ for $30 \mathrm{~min}$ at room temperature. After blocking with $1 \%$ bovine serum albumin for $1 \mathrm{~h}$, the 
cells were then incubated with E-cadherin and vimentin antibodies at $4{ }^{\circ} \mathrm{C}$ overnight. After being washed with PBS, the sections were stained with Alexa Fluor 488 dye anti-rabbit antibodies at room temperature for $1 \mathrm{~h}$. Nuclei were counterstained with $4^{\prime}, 6$-diamidino-2-phenylindole and the images were analyzed using a fluorescence microscopy (Nikon, Tokyo, Japan).

\section{RNA Isolation and Real-Time PCR}

Total RNA isolation was performed with TRIzol reagent (Takara, Dalian, China) according to the manufacturer's protocol. cDNA was synthesized with a Reverse Transcript Kit (Takara). And then real-time PCR was conducted on a quantified machine (Roche, Switzerland). All the experiments were administrated following the instructions of the kits. The primers used in our experiments are listed in Table 1.

\section{Wound-Healing Assay}

Cells were seeded in six-well plates and cultured until 30-50\% confluence. After transfection with TBK1 siRNA for $24 \mathrm{~h}$, the confluent cell monolayer was scratched with a pipette tip to produce a vertical line across the middle of the wells. The detached cells were washed out with PBS for three times. Then the cells were cultured in serum-free DMEM and treated with ${ }^{60} \mathrm{Co} \gamma$-rays. Pictures were taken immediately after the wound (at $0 \mathrm{~h}$ ) and at $40 \mathrm{~h}$ after the radiation using a microscopy (Nikon).

\section{RESULTS}

\section{Irradiation Induces Molecular Changes of A549} Associated with EMT

A549 cells were irradiated with a single dose of $2,4,6$, and 8 Gy $\gamma$-rays. Cell morphology was observed at 24,48 , and $72 \mathrm{~h}$ post irradiation, including enlargement of cell space, loss of cell polarity, the appearance of spindle-shaped cells, and increased formation of pseudopodia, which are consistent with the mesenchymal phenotype (data not shown). To evaluate if this change was the result of EMT, we tested the expression of EMT-associated proteins by western blotting. At the protein level, radiation reduced expression of the epithelial marker E-cadherin and enhanced the expression of the mesenchymal marker vimentin in a time- and dose-dependent manner compared with non-irradiated control levels (Figures $1 \mathrm{a}$ and $\mathrm{b}$ ). These data indicate that radiation induces EMT of human lung cancer cells A549. Based on these initial findings, we selected the dose of $8 \mathrm{~Gy}$ and $72 \mathrm{~h}$ of treatment for further experiments.

\section{TBK1 Inhibition Attenuates Radiation-Induced EMT}

We next determined the contribution of TBK1 signaling to radiation-induced EMT. Using TBK1 siRNAs to knockdown TBK1 levels in A549 cells, the normally observed induction of vimentin caused by radiation treatment was attenuated and the decrease in basal E-cadherin levels was prevented (Figure $2 \mathrm{a}$ ). These results were also substantiated
Table 1 PCR primers

\begin{tabular}{lll}
\hline Name & Left & Right \\
\hline TGF- $\beta$ & GCTGTCAGCTCCAAAACTCC & CGGAGCTCTGATGTGTTGAA \\
ZEB1 & CAGCTCTGGGTGGAGAAGAC & CCTGACCCACTTCCAACAGT \\
GAPDH & CGGCTACTAGCGGTITACG & AAGAAGATGCGGCTGACTGT
\end{tabular}

by immunofluorescence analysis: knockdown of TBK1 overrode the characteristic downregulation of E-cadherin (Figure 2b) and upregulation of vimentin in A549 cells (Figure 2c). Another characteristic of EMT is increased cell migration and invasion. We analyzed the migratory and invasive properties of A549 using a wound-healing assay. Consistently, the acquisition of increased migration was prevented by knockdown of TBK1 (Figure 2d). Taken together, our data suggest that TBK1 inhibition attenuates radiation-induced responses in A549 cells.

\section{TBK1 Inhibition Regulates Radiation-Induced ZEB1 Expression}

To gain further insight into the molecular mechanisms of TBK1 regulation of EMT, we aimed to identify transcription factors whose expression regulates EMT and is regulated by TBK1. ZEB1 have major regulatory roles on TGF- $\beta$-induced EMT program, which acts as an E-cadherin repressor. ${ }^{26}$ To investigate ZEB1 expression during TBK1-regulated EMT, we used the RT-PCR approach to identify whether ZEB1 expression was altered in the initial stage of EMT induced by radiation. As shown in Figure 3a, mRNA of ZEB1 was induced at $3 \mathrm{~h}$ after radiation and silencing TBK1 attenuated this change. We next performed western blot analysis with antibodies that specifically recognize ZEB1. Radiation caused a time-dependent increase in ZEB1 protein level, however, TBK1 knockdown attenuated the increasing expression of ZEB1 protein induced by radiation (Figure $3 \mathrm{~b}$ ). Thus, we next examined the association between TBK1 and ZEB1 using the overexpression plasmid of ZEB1. Our data showed that overexpression of ZEB1 overrode the role of TBK1 inhibition on radiation-induced EMT (Figure 3c). Based on the result, we viewed TBK1-regulated radiation-induced EMT via repression of ZEB1.

\section{TGF- $\beta /$ Smad Pathway Are not Attenuated by TBK1 Inhibition}

It is reported that concentrations of TGF- $\beta$ were increased in patients' serum after radiotherapy and were identified as a significant risk factor for radiotherapy. ${ }^{27}$ Numerous studies have attempted to clarify the role of TGF- $\beta$ in EMT ${ }^{28}$ and the pathways involved in radiation-induced EMT. ${ }^{18}$ Therefore, we studied the TGF- $\beta$ signaling in response to radiation and identified that if TBK1 modulated it. We first investigated the level of TGF- $\beta$ in cell lysate by real-time PCR. As shown in 
a

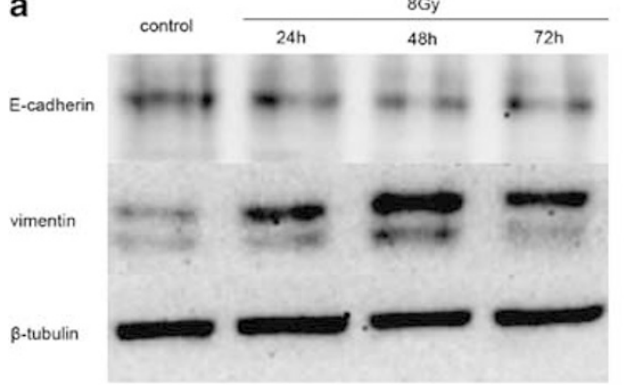

b

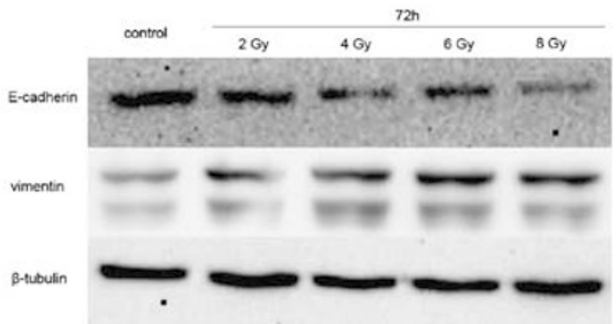

Figure 1 Irradiating A549 cells differentially modulate the expression of E-cadherin and vimentin in a time- and dose-dependent manner. (a, b) A549 cells were irradiated with different dose of ${ }^{60} \mathrm{Co} \gamma$-rays and were harvested at 24,48 , and $72 \mathrm{~h}$ post irradiation. Western blots were used to detect biomarker specific for EMT. $\beta$-Tubulin was used as loading control.

a

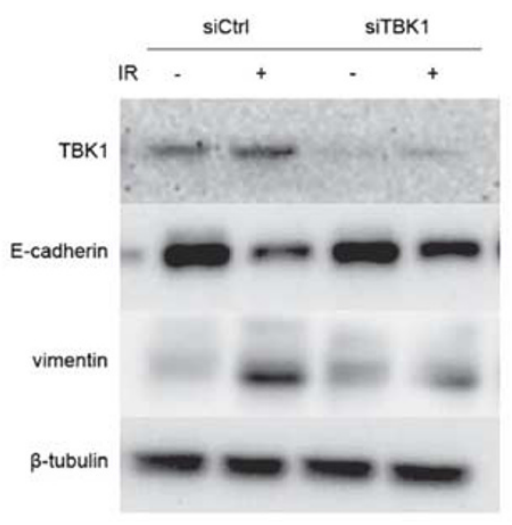

b
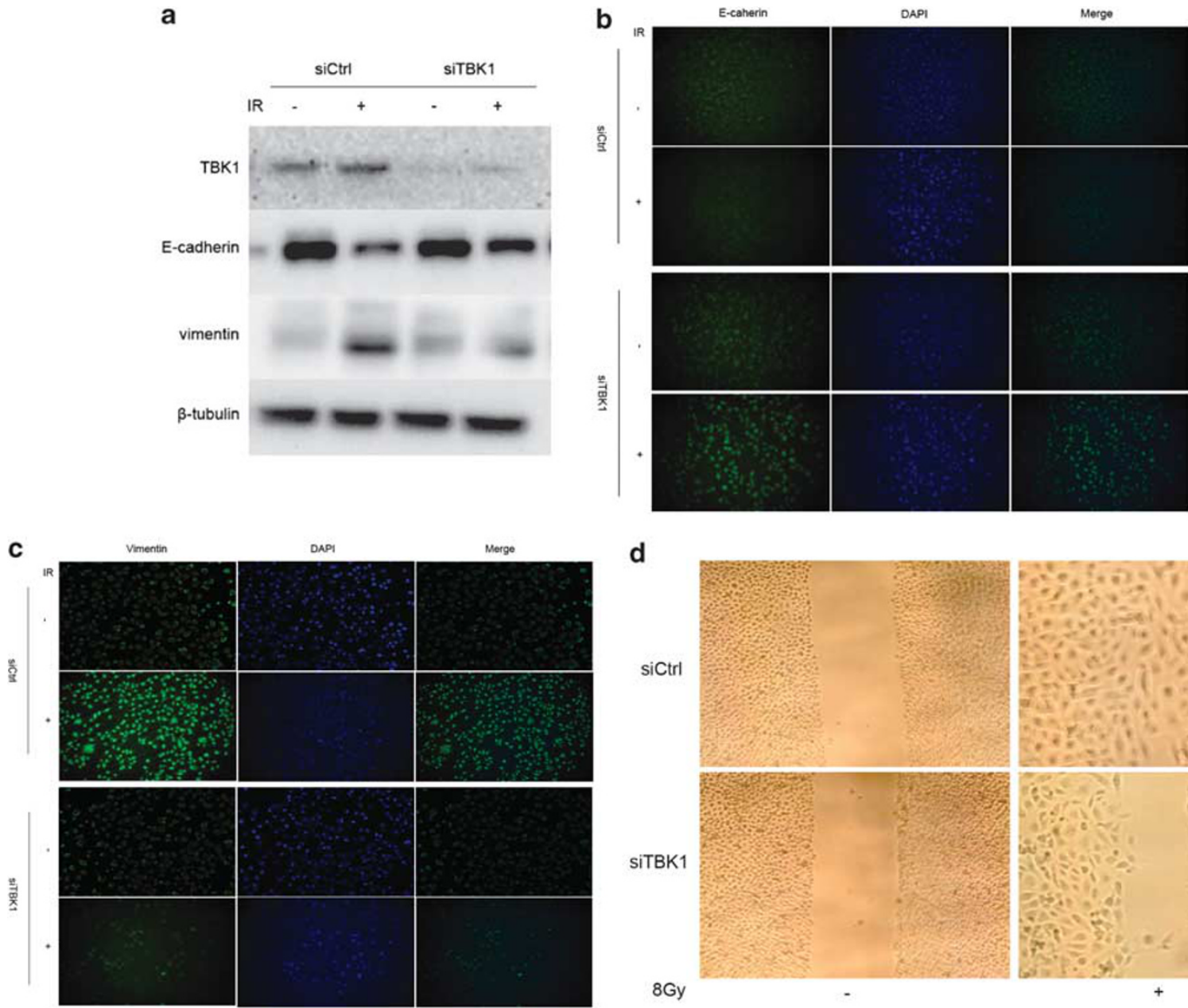

d

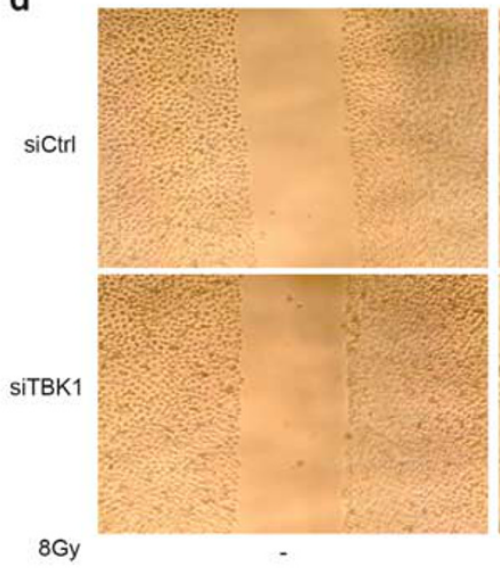

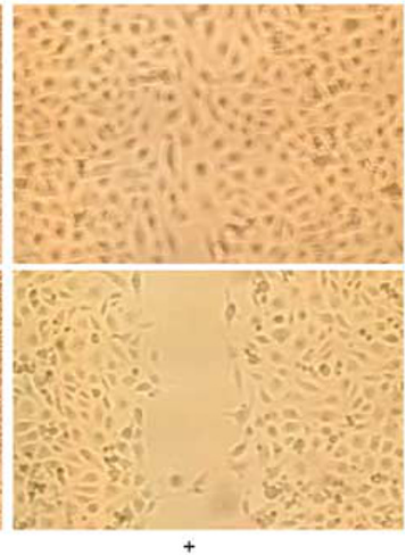

Figure 2 TBK1 inhibition attenuates radiation-induced EMT. (a) A549 cells were transiently transfected with siRNA-specific TBK1 or with control siRNA. After $24 \mathrm{~h}$, cells were irradiated with $8 \mathrm{~Gy}$ of ${ }^{60} \mathrm{Co} \gamma$-rays and were harvested at $72 \mathrm{~h}$ post irradiation. Expression of each protein was evaluated by western blot analysis. $\beta$-Tubulin was used as loading control. (b) Immunofluorescence staining for E-cadherin (green) and 4',6-diamidino-2-phenylindole (DAPI; blue) in control and treated cells at $72 \mathrm{~h}$ post irradiation (original magnification $\times 100$ ). (c) Immunofluorescence staining for vimetin (green) and DAPI (blue) in control and treated cells at $72 \mathrm{~h}$ post irradiation (original magnification $\times 100$ ). (d) A549 cells were transfected with siCtrl or siTBK1 and subjected to a wound-healing assay with radiation or vehicle. Representative images were photographed at $\times 100$ magnification right after the scratch and at $\times 400$ magnification $40 \mathrm{~h}$ after radiation.

Figure $4 \mathrm{a}$, the TGF- $\beta$ contents were enhanced in A549 after irradiation, but silencing TBK1 did not affect TGF- $\beta$. TGF- $\beta$ / Smad signaling is a classic pathway in regulating EMT, the expression of p-Smad3 in A549 was increased after irradiation, whereas the level of smad3 remained unchanged (Figure 4b). Whereas attenuation of TBK1 did not decrease 


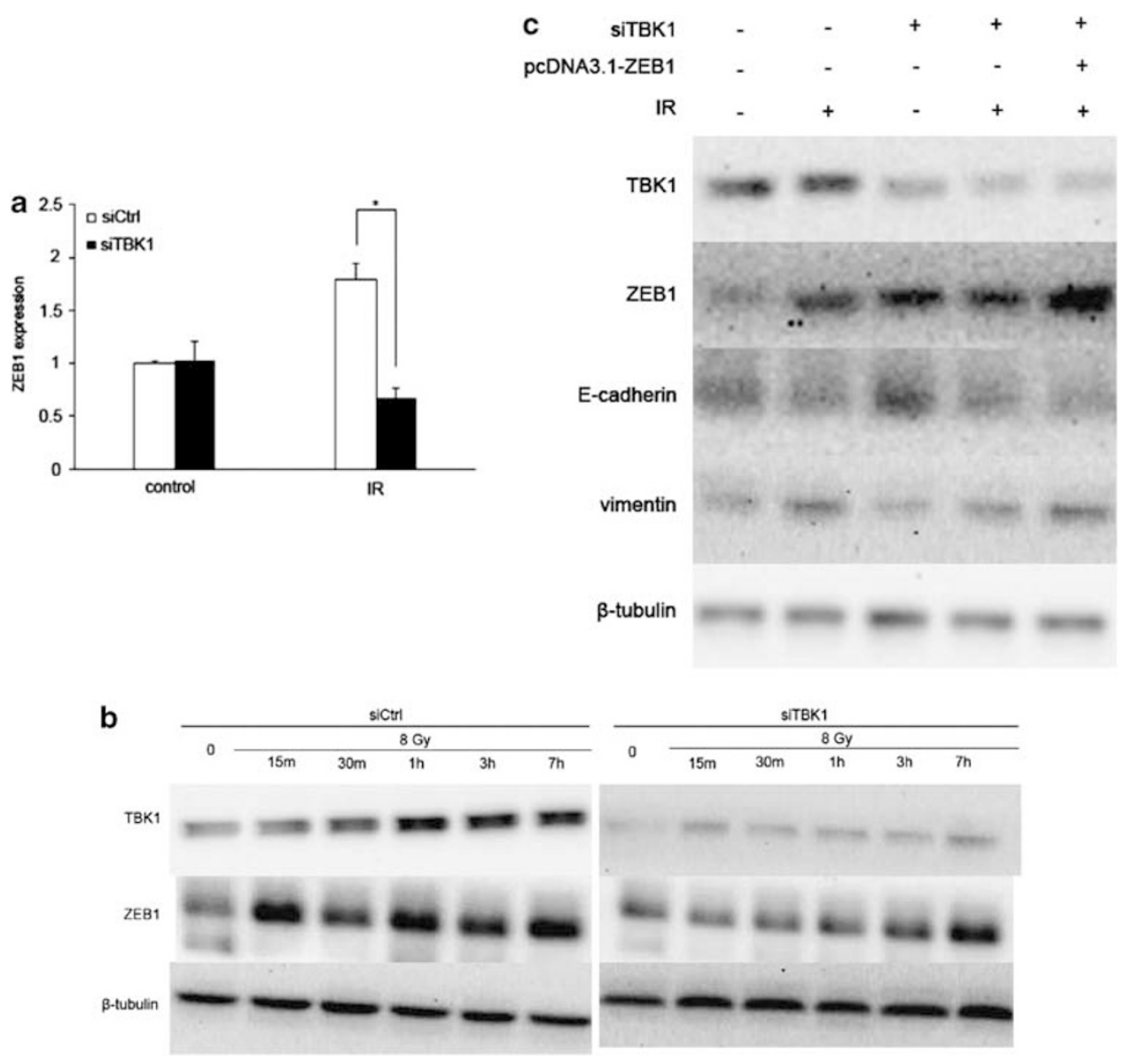

Figure 3 TBK1 inhibition regulates radiation-induced ZEB1 expression. (a) A549 cells were transfected with a control or a TBK1-specific siRNA. After $24 \mathrm{~h}$ post-transfection, cells were left untreated or treated with $8 \mathrm{~Gy}$ of ${ }^{60} \mathrm{Co} \gamma$-rays, and total RNA were collected at $3 \mathrm{~h}$ after irradiation. RT-PCR analysis was done using a primer for ZEB1. The data were reported as means \pm s.e.m., ${ }^{*} P<0.05$. (b) A549 cells were transfected with a control or a TBK1-specific siRNA. After $24 \mathrm{~h}$ post-transfection, cells were left untreated or treated with $8 \mathrm{~Gy}$ of ${ }^{60} \mathrm{Co} \gamma$-rays, and protein samples were collected at various time points after irradiation. Western blot analyses were used to detect transcription factor ZEB1. Equal protein loading was verified by the analysis of $\beta$-tubulin. (c) A549 cells were transfected with siCtrl, siTBK1, empty vector, plasmid of ZEB1, or were co-transfected with siTBK1 and plasmid of ZEB1. After $24 \mathrm{~h}$, cells were irradiated with or without $8 \mathrm{~Gy}$ of ${ }^{60} \mathrm{Co} \gamma$-rays for an additional $72 \mathrm{~h}$. EMT-specific biomarkers were detected by western blot analysis. Equal protein loading was verified by the analysis of $\beta$-tubulin.

the radiation-induced expression of $\mathrm{p}-\mathrm{Smad} 3$, indicating the minimal requirement of smad signaling in TBK1 effect (Figure $4 \mathrm{c}$ ). These data suggest that TGF- $\beta /$ Smad signaling are activated by radiation and may have a role in radiation-induced EMT, but TBK1 signaling does not regulate the phosphorylation of smad3 in A549 cells, indicating that the effects of TBK1 inhibition require distinct signaling intermediates.

\section{Activation of NF-KB Signaling Is not Required for TBK1 Inhibition}

$\mathrm{NF}-\kappa \mathrm{B}$ is a transcription factor that not only has an important role in the regulation of cell apoptosis and tumourigenesis but also is essential for EMT induction and maintenance by targeting ZEB1/2. ${ }^{29,30}$ Moreover, TBK1 participates in inflammatory pathways related to activation of NF- $\kappa \mathrm{B} .{ }^{19}$ Therefore, we studied the activation of NF- $\kappa \mathrm{B}$ signaling in response to radiation and its contribution to
TBK1 signaling. Activation of the NF- $\kappa$ B signaling pathway was determined by measuring the expression of the NF- $\kappa \mathrm{B}$ inhibitory protein inhibitor, $\kappa \mathrm{B}-\alpha(\mathrm{I} \kappa \mathrm{B}-\alpha)$ and $\mathrm{p}-\mathrm{I} \kappa \mathrm{B}-\alpha$. We found that radiation could degrade as well as phosphorylate $\mathrm{I} \kappa \mathrm{B}-\alpha$ within 3 hours, indicating that radiation may or can activate NF- $\kappa$ B pathway (Figure $5 \mathrm{a}$ ). Next, we determined the contribution of NF- $\kappa$ B signaling to the TBK1 effect. Inhibition of TBK1 by using siRNA did not affect the protein level of $\mathrm{I} \kappa \mathrm{B}-\alpha$ and $\mathrm{p}-\mathrm{I} \kappa \mathrm{B}-\alpha$ induced by radiation (Figure $5 \mathrm{~b}$ ). These findings indicate that activation of the NF- $\kappa$ B signaling pathway is not required for TBK1 inhibition on radiationinduced EMT.

TBK1 Inhibition Regulates Radiation-Induced EMT via Attenuating Radiation-Induced Changes in p-GSK-3 $\beta$

GSK-3 signaling has been demonstrated to have a modulatory effect on EMT in various cell types ${ }^{31}$ and has been 


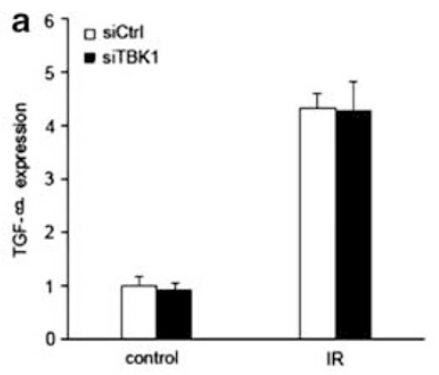

b
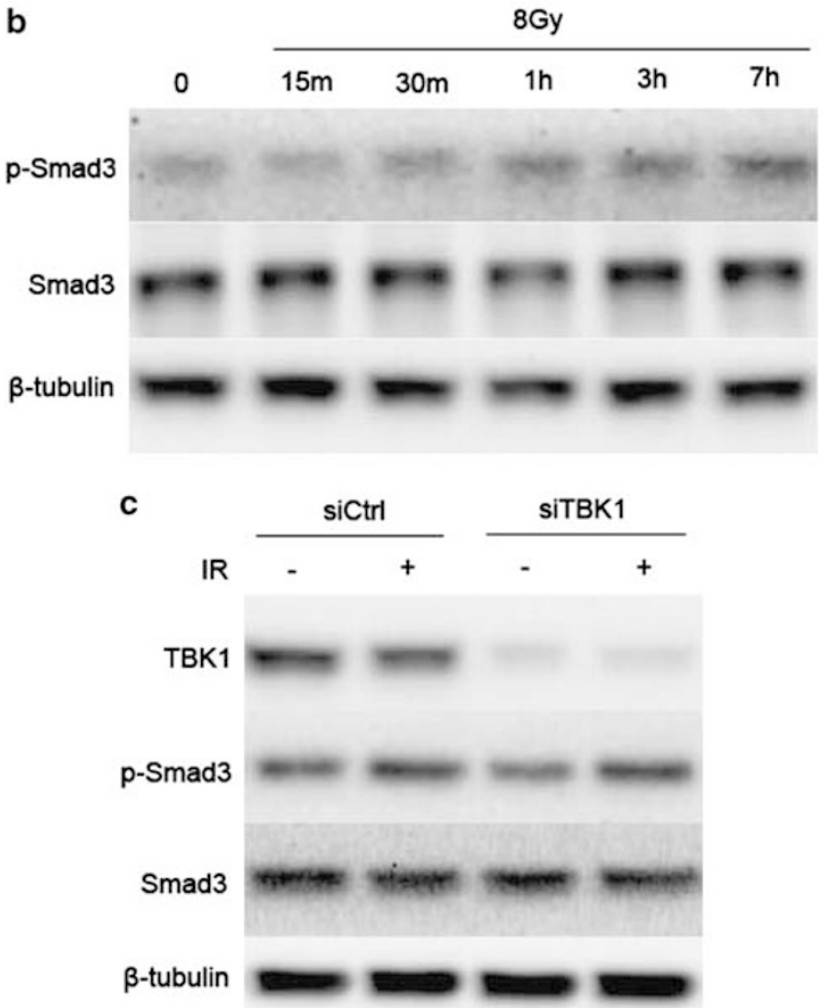

Figure 4 TGF- $\beta /$ Smad pathway is not attenuated by TBK1 inhibition. (a) A549 cells were transfected with a control or a TBK1-specific siRNA. After $24 \mathrm{~h}$ post-transfection, cells were treated with or without $8 \mathrm{~Gy}$ of ${ }^{60} \mathrm{Co} \gamma$ rays, and total RNA were collected at $72 \mathrm{~h}$ after irradiation. RT-PCR analysis was done using a primer for TGF- $\beta$. The data were reported as means \pm s.e.m., $P>0.05$. (b) A549 cells were irradiated with 8 Gy of ${ }^{60} \mathrm{Co}$ $\gamma$-rays and were harvested at various time points after irradiation. Western blot analyses were used to detect Smad3 and p-Smad3. Equal protein loading was verified by the analysis of $\beta$-tubulin. (c) A549 cells were transfected with a control or a TBK1-specific siRNA. After $24 \mathrm{~h}$ posttransfection, cells were treated with or without $8 \mathrm{~Gy}$ of ${ }^{60} \mathrm{Co} \gamma$-rays for an additional $3 \mathrm{~h}$. Western blot analyses were used to detect whether Smad3 and $\mathrm{p}$-Smad 3 were affected by TBK1 knockdown. Equal protein loading was verified by the analysis of $\beta$-tubulin.

previously implicated in certain features of radiation-induced EMT. ${ }^{23}$ We hypothesized that TBK1 may have a regulatory role in GSK-3 $\beta$ signaling. Western blot analyses showed that phosphorylation of GSK-3 $\beta$ (S9) was significantly increased in irradiated A549 cells in a time-dependent manner, whereas the levels of total GSK-3 $\beta$ remained unchanged (Figure 6a). a

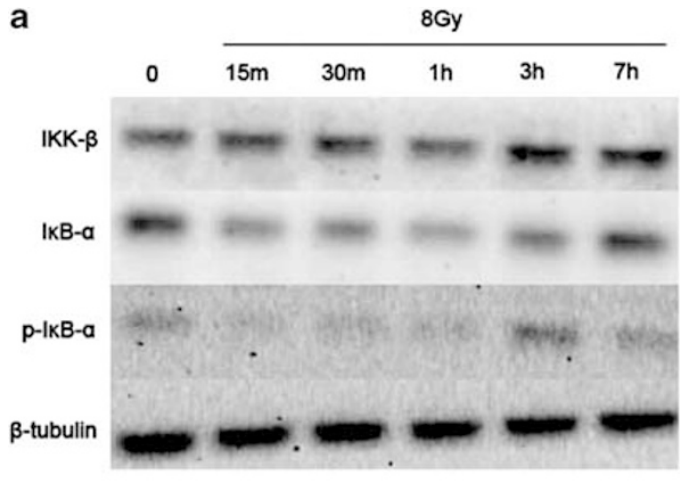

b

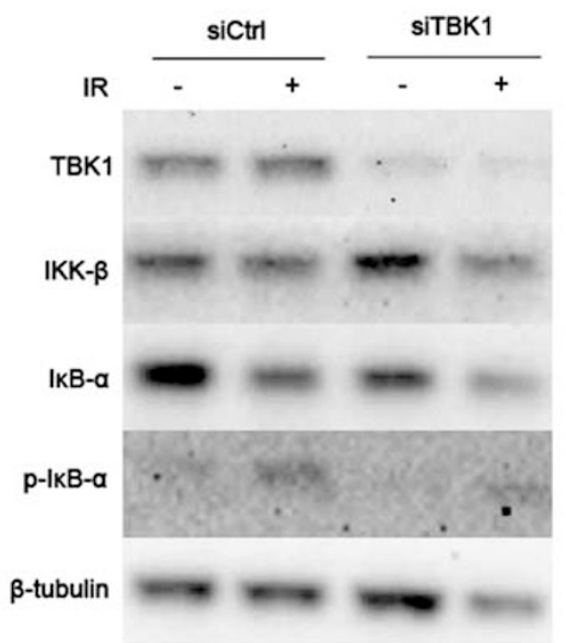

Figure 5 Activation of NF- $\kappa$ B signaling is not required for TBK1 inhibition. (a) A549 cells were irradiated with $8 \mathrm{~Gy}$ of ${ }^{60} \mathrm{Co} \gamma$-rays and were harvested at various time points after irradiation. The expression of IKK- $\beta$ and the degradation and phosphorylation of $I_{\kappa} \mathrm{B}-\alpha$ were examined by western blot analysis. $\beta$-Tubulin served as the internal control. (b) A549 cells were transfected with a control or a TBK1-specific siRNA. After $24 \mathrm{~h}$ post-transfection, cells were treated with or without $8 \mathrm{~Gy}$ of ${ }^{60} \mathrm{Co} \gamma$ rays for an additional $3 \mathrm{~h}$. Western blot analyses were used to detect whether IKK- $\beta$ and the degradation and phosphorylation of $I_{\kappa} \mathrm{B}-\alpha$ were affected by TBK1 knockdown. $\beta$-Tubulin served as the internal control.

Interestingly, we observed that inhibition of TBK1 by using siRNAs resulted in a significant decrease in phosphorylated GSK-3 $\beta$ in A549 cells, which indicated that TBK1 inhibition could overrode the effect that radiation inactivated GSK-3 $\beta$ (Figure 6a). To further investigate the role of GSK-3 $\beta$ in the regulation of radiation-induced EMT by TBK1, we used a GSK-3 $\beta$ inhibitor, SB216763, that has been shown previously to inhibit GSK-3 $\beta .^{32}$ In agreement with this finding, we found that stimulation with SB216763 attenuated the expression of biomarker specific for EMT and decreased the expression of transcription factor ZEB1 compared with only inhibition of TBK1 by using siRNAs (Figures $6 \mathrm{~b}$ and $\mathrm{c}$ ). These findings imply that GSK-3 $\beta$ signaling is capable of producing a powerful inhibitory signal for radiation-induced EMT, suggesting that phosphorylation of GSK- $3 \beta$ by TBK1 inhibition is a plausible explanation for its anti-EMT effects. 
a

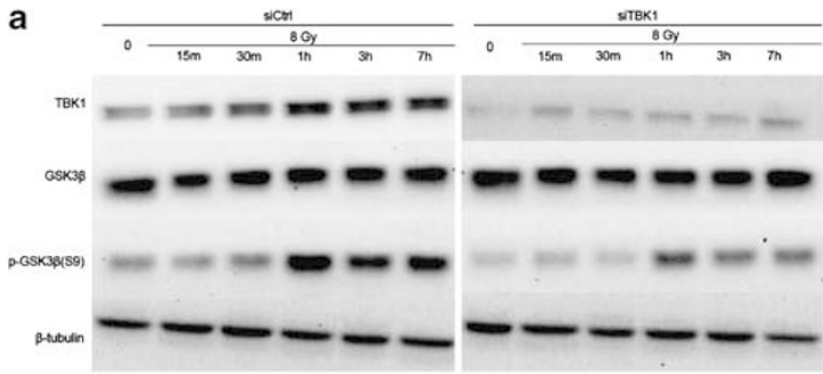

b

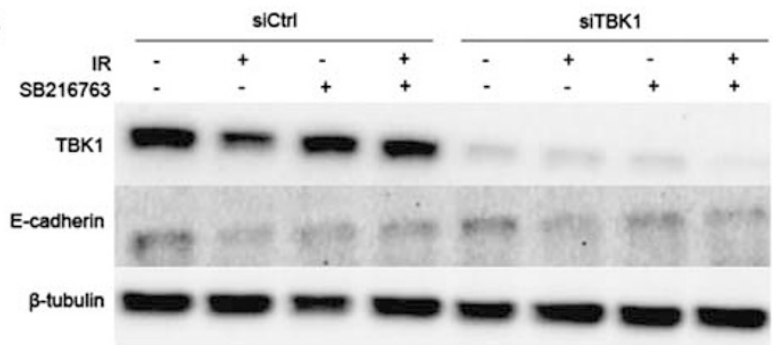

C

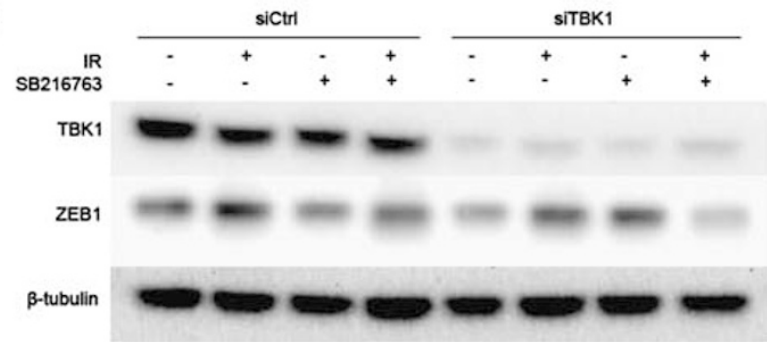

Figure 6 TBK1 inhibition regulates radiation-induced EMT via attenuating radiation-induced changes in p-GSK-3 $\beta$. (a) A549 cells were transfected with a control or a TBK1-specific siRNA. After $24 \mathrm{~h}$ post-transfection, cells were left untreated or treated with $8 \mathrm{~Gy}$ of ${ }^{60} \mathrm{Co} \gamma$-rays, and protein samples were collected at various time points after irradiation. Western blot analyses were used to detect GSK-3 $\beta$ and p-GSK-3 $\beta$. $\beta$-Tubulin served as the internal control. (b, c) A549 cells were transfected with a control or a TBK1-specific siRNA. After $24 \mathrm{~h}$ post-transfection, the cells were treated with $10 \mu \mathrm{M} \mathrm{SB} 216763$ for $16 \mathrm{~h}$, irradiated with $8 \mathrm{~Gy}$ of ${ }^{60} \mathrm{Co} \gamma$-rays, and protein samples were collected at various time points after irradiation. Expression of the epithelial marker E-cadherin and transcription factor ZEB1 was detected by western blot analysis. $\beta$-Tubulin served as the internal control.

\section{DISCUSSION}

Radiation is a double-edged sword: it not only kills tumor cells, but can also lead to development of distant metastases, induce radioresistance, and damage normal tissues. Recently, EMT has been found to have malignant characteristics in confer cancer cells, causing the cells to have a propensity for metastatic dissemination, resistance to various therapies, and recurrence. Accumulating evidence indicates that radiation is one of the inducers of EMT, so understanding the relationship between radiation and EMT and generating novel targets that can fight cancer progression as well as normal tissue injuries induced by radiotherapy are necessary.

TBK1, an atypical I $\kappa$ B kinase family member, has been defined as a central molecule in cell regulatory networks responsive to inflammatory cytokines and pathogen surveil- lance receptors. ${ }^{33}$ Chien et al showed that Ralb GTPase regulated TBK1, provided an unexpected link between the signaling pathways that promote inflammation and cancer. ${ }^{34}$ In tumor cells the RalB/TBK1 pathway inhibits apoptosis and in nontumorigenic cells it stimulates the innate immune response. $^{35}$ Emerging evidences suggest that targeting TBK1 may be therapeutically beneficial for the treatment of certain tumors. But the role of TBK1 in radiation is still unclear. Our previous study showed that TBK1 knockdown enhanced radiation-induced death and repressed proliferation in MDAMB-231 cells. The expression level of TBK1 in MDA-MB-231 was higher than in MCF-7, suggesting that TBK1 may contribute to malignant characteristics. ${ }^{21}$ Based on these findings, we viewed TBK1 as an oncogene-like kinase under the influence of radiation. We have also reported that TBK1 is a downstream effector of the miR-200c-driven pathway, ${ }^{21}$ which inhibits EMT by directly targeting ZEB1 and ZEB2. ${ }^{22}$ In the present study, we used the A549 model of radiation induction of EMT to investigate the effect of TBK1 expression on EMT. Here we reported for the first time that downregulation of TBK1 expression in A549 cells results in a loss of expression of epithelial markers along with a gain in expression of mesenchymal markers. Moreover, the cells show the increasing migrative ability as assessed by a woundhealing assay. Taken together, the results point to the regulation of endogenous $\mathrm{TBK} 1$ protein expression as a potential strategy to target EMT and related invasive behavior in cancer cells.

The most significant finding in our study was that TBK1 acted as a negative regulator of radiation-induced ZEB1dependent EMT. It is well documented that ZEB1 is an important transcript factor of EMT by inhibiting an E-box gene, such as E-cadherin. ${ }^{36}$ Several previous studies suggest that ectopic expression of miR-200c hinders progression of EMT in TGF- $\beta$-treated cells by downregulation of $\mathrm{ZEB1}^{37}$ and miR-200c induced endothelial cell apoptosis and senescence via ZEB1 inhibition. ${ }^{38} \mathrm{ZEB} 1$ is induced by TGF$\beta$ during smooth muscle differentiation, in which it interacts with Smad3 and SRF to transactivate the genes encoding smooth muscle $\alpha$-actin and smooth muscle myosin heavy chain. ${ }^{39}$ Furthermore, overexpression of ZEB1 in MCF-10A cells reduced E-cadherin and p65 expression and displayed an EMT phenotype. ${ }^{31}$ In our present study, we show that TBK1 can attenuate radiation-induced upregulation of ZEB1. We believe ZEB1 to be the target of TBK1 inhibition.

The intensive investigation of molecular and cellular changes that can regulate EMT is focused on TGF- $\beta$. TGF- $\beta$ is a multifunctional cytokine that regulates various cellular functions via diverse signaling pathways. Stimulation of epithelial cells with TGF- $\beta$ is the classical way to induce EMT. However, Smad signaling is activated in response to TGF- $\beta$ and leads to EMT. It has been shown that IR can activate TGF- $\beta$ signaling, ${ }^{40}$ and EMT mediated by TGF- $\beta /$ Smad signaling may be a mechanism in the IR-promoted migration and invasion of cancer cells. ${ }^{18}$ Consistent with the results of 
previous researchers, we found an increase in TGF- $\beta$ and phosphorylation of Smad3, but we did not detect such changes in TBK1 knockdown cells. This suggests the lack of cross-talk between TBK1 signaling and the TGF- $\beta / \mathrm{Smad}$ pathway. Other research showed that low-dose radiationinduced EMT through NF- $\kappa \mathrm{B}$ p65 signaling enhanced the migration and invasion of cervical cancer cells, and when NF- $\kappa \mathrm{B}$ p65 was silenced, the EMT process was reversed. To explore the underlying mechanism of TBK1, we investigated the NF- $\kappa \mathrm{B}$ signaling pathway. TBK1 mediates the inducible phosphorylation of p65 at Ser-536, ${ }^{41}$ and upregulation of TBK1 is proposed to contribute to the activation of NF- $\kappa$ B. ${ }^{42}$ Our data show that IR activated NF$\kappa \mathrm{B}$, which led to the phosphorylation and degradation of $\mathrm{I} \kappa \mathrm{B}-\alpha$. However, inhibition of TBK1 did not suppress NF- $\kappa \mathrm{B}$.

GSK- $3 \beta$ is a ubiquitous and promiscuous kinase that participates in several key signaling pathways that can have a role in EMT, such as Wnt, Hedgehog, TGF- $\beta$, phosphatidylinositol-3'-kinase, and Notch pathways. ${ }^{32}$ In many cells, GSK- $3 \beta$ is constitutively active and can phosphorylate Snail to facilitate its degradation. GSK-3 $\beta$ can be inactivated by phosphorylation at its N-terminal serine 9 residue. Inactivation of GSK$3 \beta$ stabilizes Snail and succumbs to EMT induction. ${ }^{43}$ In the current study, western blot analyses showed that radiation increased the phosphorylation of GSK- $3 \beta$ at serine 9 residue and silencing TBK1 can obviously attenuates the phosphorylation, suggesting that inhibition of TBK1 has been linked with activation of GSK-3 $\beta$. Thus, we hypothesize that TBK1 inhibition can attenuate radiation-induced expression of ZEB1 via activation of the GSK-3 $\beta$. In support of this hypothesis, pretreatment of cells with the GSK- $3 \beta$ inhibitor SB216763 promotes TBK1 inhibition, accelerating the upregulation of E-cadherin and downregulation of ZEB1. Taken together, our data suggest that GSK- $3 \beta$ is necessary for TBK1 signaling in radiation-induced EMT.

Based on our findings, we have shown that TBK1 inhibition in A549 cells is likely to contribute significantly to attenuation of radiation-induced EMT. Mechanistically, a pathway involving activated GSK-3 $\beta$ and decreased transcription factor ZEB1 is implicated. Taken together, increasing understanding of the role TBK1 in radiationinduced EMT will not only provide new insight into cancer research, but also pave the way for more effective treatments in radiotherapy.

\section{ACKNOWLEDGMENTS}

This work was supported by the grants from National Natural Science Foundation of China (No. 31370840)

\section{DISCLOSURE/CONFLICT OF INTEREST}

The authors declare no conflict of interest.

1. Jemal A, Forman D. 'Global cancer statistics,'. CA-Cancer J Clin 2011;61:69-90.

2. Jemal A, Siegel R, Ward E, et al. 'Cancer statistics, 2008,'. CA-Cancer J Clin 2008;58:71-96.
3. She J, Yang P, Hong Q, et al. Lung cancer in China: challenges and interventions. Chest 2013;143:1117-1126.

4. Blackstock AW, Govindan R. Definitive chemoradiation for the treatment of locally advanced non small-cell lung cancer. J Clin Oncol 2007;25:4146-4152.

5. Salama JK, Vokes EE. New radiotherapy and chemoradiotherapy approaches for non-small-cell lung cancer. J Clin Oncol 2013;31: 1029-1038.

6. Barcellos-Hoff MH, Park C, Wright EG. Radiation and the microenvironment-tumorigenesis and therapy. Nat Rev Cancer 2005; 5:867-875.

7. Madani I, De Neve W, Mareel M. Does ionizing radiation stimulate cancer invasion and metastasis? Bull Cancer 2008;95:292-300.

8. McBride $\mathrm{WH}$, Chiang $\mathrm{CS}$, Olson $\mathrm{JL}$, et al. A sense of danger from radiation. Radiat Res 2004;162:1-19.

9. Blackhall F, Faivre-Finn C. Treatment of limited small cell lung cancer: an old or new challenge? Curr Opin Oncol 2011;23:158-162.

10. Kawabe $\mathrm{T}$, Phi $\mathrm{JH}$, Yamamoto $\mathrm{M}$, et al. Treatment of brain metastasis from lung cancer. Prog Neurol Surg 2012;25:148-155.

11. Chambers AF, Groom AC, MacDonald IC. Dissemination and growth of cancer cells in metastatic sites. Nat Rev Cancer 2002;2:563-572.

12. Eccles SA, Welch DR. Metastasis: recent discoveries and novel treatment strategies. Lancet 2007;369:1742-1757.

13. Kalluri $R$, Weinberg RA. The basics of epithelial-mesenchymal transition. J Clin Invest 2009;119:1420-1428.

14. Turley EA, Price MA, Li GY, et al. Mechanisms of disease: epithelialmesenchymal transition-does cellular plasticity fuel neoplastic progression? Nat Clin Pract Oncol 2008;5:280-290.

15. Sato M, Shames DS, Hasegawa Y. Emerging evidence of epithelialmesenchymal transition in lung carcinogenesis. Respirology 2012;17: 1048-1059.

16. Yan $S$, Wang $Y$, Yang $Q$, et al. Low-dose radiation-induced epithelialmesenchymal transition through $\mathrm{NF}-\kappa \mathrm{B}$ in cervical cancer cells. Int $J$ Oncol 2013;33:938-946.

17. Kawamoto A, Yokoe $T$, Tanaka $K$, et al. Radiation induces epithelialmesenchymal transition in colorectal cancer cells. Oncol Rep 2012; 27:51-57.

18. Zhou YC, Liu JY, Li J, et al. lonizing radiation promotes migration and invasion of cancer cells through transforming growth factor-betamediated epithelial-mesenchymal transition. Int J Radiat Oncol Biol Phys 2011;81:1530-1537.

19. Pomerantz JL, Baltimore D. NF kappaB activation by a signaling complex containing TRAF2, TANK and TBK1, a novel IKK-related kinase. EMBO J 1999;18:6694-6704.

20. Zhang $M$, Wang $L$, Zhao $X$, et al. TRAF-interacting protein (TRIP) negatively regulates IFN- $\beta$ production and antiviral response by promoting proteasomal degradation of TANK-binding kinase 1. J Exp Med 2012;209:1703-1711.

21. Lin J, Liu C, Gao F, et al. MiR-200c enhances radiosensitivity of human breast cancer cells. J Cell Biochem 2013;114:606-615.

22. Hurteau GJ, Carlson JA, Spivack SD, et al. Overexpression of the microRNA hsa-miR-200c leads to reduced expression of transcription factor 8 and increased expression of E-cadherin. Cancer Res 2007;67: 7972-7976.

23. Nagarajan D, Melo $T$, Deng $Z$, et al. ERK/GSK3 $\beta /$ Snail signaling mediates radiation-induced alveolar epithelial-to-mesenchymal transition. Free Radical Biol Med 2012;52:983-992.

24. Thotala DK, Hallahan DE, Yazlovitskaya EM. Inhibition of glycogen synthase kinase 3 beta attenuates neurocognitive dysfunction resulting from cranial irradiation. Cancer Res 2008;68:5859-5868.

25. Thotala DK, Hallahan DE, Yazlovitskaya EM. Glycogen synthase kinase $3 \beta$ inhibitors protect hippocampal neurons from radiation-induced apoptosis by regulating MDM2-p53 pathway. Cell Death Differ 2012; 19:387-396.

26. Bracken CP, Gregory PA, Kolesnikoff N, et al. A double-negative feedback loop between ZEB1-SIP1 and the microRNA-200 family regulates epithelial-mesenchymal transition. Cancer Res 2008;68:7846-7854.

27. Zhang XJ, Sun JG, Sun J, et al. Prediction of radiation pneumonitis in lung cancer patients: a systematic review. J Cancer Res Clin Oncol 2012;138:2103-2116.

28. Fuxe J, Karlsson MC. TGF- $\beta$-induced epithelial-mesenchymal transition: a link between cancer and inflammation. Semin Cancer Biol 2012; 22:455-461. 
29. Huber MA, Azoitei N, Baumann B, et al. NF-kappaB is essential for epithelial-mesenchymal transition and metastasis in a model of breast cancer progression. J Clin Invest 2004;114:569-581.

30. Chua HL, Bhat-Nakshatri P, Clare SE, et al. NF-kappaB represses E-cadherin expression and enhances epithelial to mesenchymal transition of mammary epithelial cells: potential involvement of ZEB1 and ZEB2. Oncogene 2007;26:711-724.

31. Doble BW, Woodgett JR. Role of glycogen synthase kinase-3 in cell fate and epithelial-mesenchymal transitions. Cells Tissues Organs 2007;185:73-84

32. Baarsma HA, Engelbertink LH, van Hees LJ, et al. Glycogen synthase kinase-3(GSK-3) regulates TGF- $\beta$-induced differentiation of pulmonary fibroblasts. Br J Pharmacol 2013;169:590-603.

33. Fitzgerald KA, McWhirter SM, Faia KL, et al. IKK epsilon and TBK1 are essential components of the IRF3 signaling pathway. Nat Immunol 2003;4:491-496.

34. Chien Y, Kim S, Bumeister R, et al. RalB GTPase-mediated activation of the IkappaB family kinase TBK1 couples innate immune signaling to tumorcell survival. Cell 2006;127:157-170.

35. Mantovani A, Balkwill F. RalB signaling: A bridge between inflammation and cancer. Cell 2006;127:42-44.

36. Comijn J, Berx G, Vermassen P, et al. The two-handed E box binding zinc finger protein SIP1 downregulates E-cadherin and induces invasion. Mol Cell 2001;7:1267-1278.

37. Xiong $M$, Jiang $L$, Zhou $Y$, et al. The miR-200 family regulates TGF- $\beta$-induced renal tubular epithelial to mesenchymal transition through Smad pathway by targeting ZEB1 and ZEB2 expression. Am J Physiol Renal Physiol 2011;302:F369-F379.

38. Magenta A, Cencioni C, Fasanaro P, et al. miR-200c is upregulated by oxidative stress and induces endothelial cell apoptosis and senescence via ZEB1 inhibition. Cell Death Dis 2011;18: 1628-1639.

39. Nishimura G, Manabe I, Tsushima K, et al. DeltaEF1 mediates TGF-b signaling in vascular smooth muscle cell differentiation. Dev Cell 2006;11:93-104.

40. Dancea HC, Shareef MM. Ahmed MM. Role of radiation-induced TGF-beta signaling incancer therapy. Mol Cell Pharmacol 2009;1: 44-56.

41. Buss $H$, Dorrie A, Schmitz ML, et al. Constitutive and interleukin1-inducible phosphorylation of p65NF-\{kappa\}B at serine 536 is mediated by multiple protein kinases including I\{kappa\}B kinase(IKK)\{alpha\},IKK\{beta\},IKK\{epsilon\},TRAF family member-associated (TANK)bindingkinase1(TBK1), and an unknown kinase and couples p65 to TATA-binding protein-associated factorll31-mediated interleukin-8 transcription. J Biol Chem 2004;279:55633-55643.

42. Kim HR, Lee $\mathrm{SH}$, Jung $\mathrm{G}$. The hepatitis $B$ viral $X$ protein activates NF- $\kappa \mathrm{B}$ signaling pathway through the up-regulation of TBK1. FEBS Lett 2010;584:525-530.

43. Zhou BP, Deng J, Xia W, et al. Dual regulation of Snail by GSK-3 $\beta$ mediated phosphorylation in control of epithelial-mesenchymal transition. Nat Cell Biol 2004;6:931-940. 